\title{
Multicast Throughput Order of Network Coding in Wireless Ad-hoc Networks
}

\author{
Shirish Karande ${ }^{\dagger}$, Zheng Wang ${ }^{\dagger}$, Hamid R. Sadjadpour ${ }^{\dagger}$, J.J. Garcia-Luna-Aceves ${ }^{\ddagger}$ \\ Department of Electrical Engineering ${ }^{\dagger}$ and Computer Engineering $\ddagger$ \\ University of California, Santa Cruz, 1156 High Street, Santa Cruz, CA 95064, USA \\ $\ddagger$ Palo Alto Research Center (PARC), 3333 Coyote Hill Road, Palo Alto, CA 94304, USA \\ Email:\{karandes,wzgold, hamid, jj\}@ soe.ucsc.edu
}

\begin{abstract}
We study the contribution of network coding (NC) in improving the multicast capacity of random wireless ad hoc networks. We consider a network with $n$ nodes distributed uniformly in a unit square, with each node acting as a source for independent information to be sent to a multicast group consisting of $m$ randomly chosen destinations. We show that in the presence of $\mathrm{NC}$, the per-session capacity under the protocol model has a tight bound of $\Theta\left(\frac{1}{\sqrt{m n \log (n)}}\right)$ when $m=O\left(\frac{n}{\log (n)}\right)$ and $\Theta\left(\frac{1}{n}\right)$ when $m=\Omega\left(\frac{n}{\log (n)}\right)$. Furthermore, we consider the physical model, and show that the per-session capacity has a tight bound of $\Theta\left(\frac{1}{\sqrt{m n}}\right)$ when $m=O\left(\frac{n}{(\log (n))^{3}}\right)$, and $\Theta\left(\frac{1}{n}\right)$ when $m=\Omega\left(\frac{n}{\log (n)}\right)$. Prior work has shown that these same order bounds are achievable on the basis of pure routing, which utilizes only traditional store and forward methods.
\end{abstract}

\section{INTRODUCTION}

The concept of network coding was first introduced by Yeung et. al. [1] and subsequently generalized by Ahlswede et. al. [2] for a single source multicast in arbitrary directed graphs. Since then, the interest in network coding has increased rapidly. A large number of studies have investigated the utility of network coding (NC) for wireless networks, and widely cited experiments [3], [4] have been reported in which NC has been used successfully in combination with other mechanisms to attain large throughput gains compared to approaches based on conventional protocol stacks. These results have led many to believe that a combination of NC with wireless broadcasting can lead to significant improvements in the order throughput of wireless networks. Understandably, there is significant interest in identifying the true impact of $\mathrm{NC}$ on the throughput order of wireless networks. However, the exact characterization of network capacity with NC in the presence of multiple access interference is a very hard problem, even for simple networks, and limited results have been reported to date on the subject.

Recent work [5]-[7] has shown that the throughput gain due to the use of NC in a wireless network is bounded by a constant when the traffic in the network consists of multiple unicast sessions. However, the motivation for the original work by Ahlswede et. al [2] was improving network performance for multicasting, not unicasting. Furthermore, many commercial and defense applications, such as video conferencing, require multicasting of large amounts of information, and the study of the multicast capacity of wireless ad hoc networks is an important research topic in its own right.

Several works [8]-[15] have studied the multicast and broadcast capacity of wireless networks under conventional routing, and these results show that broadcasting and multicasting significantly alter the throughput order of wireless networks. In light of these findings, the importance of multicasting and broadcasting, and recent practical results on $\mathrm{NC}$, it is natural to inquire whether the introduction of $\mathrm{NC}$ can improve the throughput order capacity of multi-source multicasting. In this work, we undertake the characterization of the multicast and broadcast throughput order of wireless ad-hoc networks in presence of network coding, which has been an open problem for the past 10 years.

We consider a network consisting of $n$ nodes distributed randomly in the network space, with each node acting as a multicast source of a group of $m$ randomly chosen nodes in the network.

The first contribution of this paper is to show that, under the protocol model, the per-session multicast capacity of random wireless ad hoc network in the presence of arbitrary $\mathrm{NC}{ }^{1}$ has a tight bound of $\Theta\left(\frac{1}{\sqrt{m n \log (n)}}\right)$ when $m=O\left(\frac{n}{\log (n)}\right)$ and $\Theta\left(\frac{1}{n}\right)$ when $m=\Omega\left(\frac{n}{\log (n)}\right)$. The second contribution of this paper is to show that, under the physical model, the persession multicast capacity of random wireless ad hoc network with arbitrary NC has a tight bound of $\Theta\left(\frac{1}{\sqrt{m n}}\right)$ when $m=$ $O\left(\frac{n}{\log (n)^{2}}\right)$, and $\Theta\left(\frac{1}{n}\right)$ when $m=\Omega\left(\frac{n}{\log (n)^{2}}\right)$.

It has already been established in the literature that the above bounds are achievable on the basis of traditional store-and-forward routing methods. Consequently, our analysis demonstrates that the throughout gain due to NC for mutlicasting and broadcasting is bounded by a constant factor!

The remainder of this paper is organized as follows. Section II surveys relevant prior work. Section III describes the network models and other concepts used in proofs. Section IV deduces the capacity results under the protocol model, and Section V addresses the physical model. Section VI

\footnotetext{
${ }^{1}$ Arbitrary NC implies that an transmitted symbol can be an arbitrary function (and not necessarily a linear function) of all the symbols recieved and generated at a node
} 
summarizes our conclusions.

\section{LiterATURE REVIEW}

Gupta and Kumar's original work focused on the unicast capacity of wireless networks [16], and many subsequent contributions have been made on the capacity of wireless networks subject to unicast traffic. However, the focus of this paper is on the capacity of wireless networks under broadcast and multicast traffic.

Tavli [8] was the first to show that $\Theta\left(n^{-1}\right)$ is a bound on the per-node broadcast capacity of arbitrary networks. Zheng [9] derived the broadcast capacity of power-constrained networks, together with another quantity called "information diffusion rate." The work by Keshavarz et al. [10] is perhaps the most general work on the computation of the broadcast capacity for any number of sources in the network.

Several efforts have addressed the multicast capacity of wireless networks, primarily under the protocol model. Jacquet and Rodolakis, [11] proved that the scaling of multicast capacity is decreased by a factor of $O(\sqrt{m})$ compared to the unicast capacity result by Gupta and Kumar [16]. This result implies that the gain attained with multicasting over transmitting the same information to each of the $m$ multicast receivers as unicasts is at least $\Theta(\sqrt{m})$. The work by Shakkottai et al [12] assumes there are $n^{\epsilon}$ multicast sources and $n^{1-\epsilon}$ destinations per flow for some $\epsilon>0$. The results from this work are limited in scope, because of its constraints on the number of sources and destinations. Li et al. [13] compute the capacity of wireless ad hoc networks for unicast, multicast, and broadcast applications. Zheng et. al. [14] independently generalized this work and introduced $(n, m, k)$-casting as a framework for the characterization of all types of information dissemination in wireless networks. Keshavarz et. al. [15] studied the multicast and broadcast capacity of wireless networks, considered the physical model, and generalized the work in [17] to the multicast regime. Recently $\mathrm{Li}$ et. al. reported results on multicast capacity under a Gaussian Channel model [18]. For $n$ sources, the throughput order reported for the Gaussian Channel model [18] is identical to that of the Physical model [13], [14]. This prior work has only addressed conventional store-and forward routing for multicast and broadcast traffic.

Ahlswede et. al. [2] showed that NC can achieve the mincut bound for a single source multicast on a directed graph. Since then, a number of theoretical results have been reported for NC. We mention a select few, which provide bounds on the NC gain over routing and/or provide max-flow min-cut type inequalities that can be used to provide outer-bounds on the rate region under NC. Li et. al [19], [20] have studied the benefits of $\mathrm{NC}$ in undirected networks. The result shows that, for a single unicast or broadcast session, there is no throughput improvement due to NC. In the case of a single multicast session, such an improvement is bounded by a factor of two. $\mathrm{S}$. Borade [21] used the classical multi-terminal cut-set bounds [22] to derive edge-cut outer bounds on the rate region under NC for multi-source unicast and multicast. Subsequent studies [23], [24] have shown that the (vertex) cut-set bounds are not tight and improved bounds can be obtained by employing more sophisticated edge-cuts. Studies such as [21], [23], [24] do not readily capture the geometric constraints of multi-hop communication in wireless ad-hoc networks. Nevertheless, there have been prior works on determining the unicast throughput order in wireless networks under NC. Liu et. al. [5], [6] have shown that the $\mathrm{NC}$ for unicast traffic in a random network (i.e., a network in which the nodes are distributed randomly in an Euclidean space and the sources and destinations are also placed randomly) is bounded by a constant factor. Keshavarz et. al. [7] extended these conclusions to arbitrary networks and an arbitrary unicast traffic pattern. To the best of our knowledge, with the exception of our own work [25], no results have been reported on the multicast throughput order of wireless ad-hoc networks in presence of network coding.

Physical network coding (PNC) [26] and analog network coding (ANC) [27] have been proposed recently, which combine $\mathrm{NC}$ with advanced processing at the physical layer that allows receivers to decode multiple concurrent transmissions. ANC was shown [27] to provide throughput gains when compared with digital network coding (i.e., receivers decode at most one packet at a time) and traditional routing (i.e., no NC and receivers decode at most one packet at a time) operating in simple network topologies in which ideal scheduling (i.e., no MAI) is assumed for channel access. Throughput gains have also been reported for PNC in simple topologies [26]. However, we have shown that the order throughput of a wireless network can be increased by embracing interference at the physical layer through multi-packet transmission (MPT) or reception (MPR), without the use of NC [28], [29]. Furthermore, we have also shown [25] that using NC together with MPT and MPR does not increase the order throughput of a wireless network for multicasting compared to what MPR and MPT can provide by themsleves.

It is clear from the above that prior work has not determined whether NC by itself can provide any gains on the multicast throughput order in wireless networks. The work presented in the rest of this paper differs from our own recent results [25] in three important ways. In our previous work [25], the sinks associated with each multicast source are bounded by a constant, whereas in this paper the number of sinks is a function of network size $n$. In our previous work [25], the models assume that a node is capable of MPT and MPR (i.e., recieving or transmitting distinct information from multiple transmitters to multiple recievers at the same time), whereas this paper assumes single packet transmission/reception. Lastly, our previous work [25] does not present any results for an SINR model, while this paper addresses the Physical Model.

\section{PRELIMINARIES}

For a continuous region $A$, we use $|A|$ to denote its area. We denote the cardinality of a set $\mathbb{S}$ by $|\mathbb{S}|$, and by $\left\|X_{i}-X_{j}\right\|$ the distance between nodes $i$ and $j$. Whenever convenient, we utilize the indicator function $1_{\{P\}}$, which is equal to one if $P$ is true and zero if $P$ is false. $\operatorname{Pr}(E)$ represents the probability of event $E$. We say that an event $E$ occurs with high probability 
(w.h.p.) if $\operatorname{Pr}(E)>(1-(1 / n))$ as $n \rightarrow \infty$. We employ the standard order notations $O, \Omega$, and $\Theta$.

We assume that the topology of a network is described by a uniformly random distribution of $n$ nodes in a unit square. Let $V=1, \ldots, n$ represent the node-set and let $X_{i}$ be the location of node $i \in V$. To avoid boundary effects, it is typical to assume that the network surface is placed upon a toroid or sphere. However, for mathematical convenience, in this work we ignore edge effects and thus assume that the network is placed in a 2-D plane. Further, in our model, as $n$ goes to infinity, the density of the network also goes to infinity. Therefore, our analysis is applicable only to dense networks. We do not consider mobility of nodes and assume a static stationary distribution of nodes. Our capacity analysis is based on both the protocol model and the physical model introduced by Gupta and Kumar [16].

Definition 3.1: The Protocol Model

We assume that all nodes use an identical transmission range $r(n)$ for all their communication. Node $i$ can successfully transmit to node $j$ if for any node $k \neq i$, that transmits at the same time as $i$ it is true that $\left|X_{i}-X_{j}\right| \leq r(n)$ and $\left|X_{k}-X_{j}\right| \geq(1+\Delta) r(n)$.

We shall utilize the following well known property [30] in our analysis

Lemma 3.2: Connectivity Criteria

For a random distribution of $n$ nodes in a unit-square, the network connectivity under the protocol model can be guaranteed w.h.p if and only if (iff)

$$
r(n) \geq r_{c}(n)=\sqrt{\frac{3 \log (n)}{n}} .
$$

\section{Definition 3.3: The Physical Model}

All transmissions at all nodes utilize an identical transmission power $P$. Node $i$ can successfully transmit to node $j$ iff the signal-to-interference/noise ratio (SINR) satisfies

$$
\operatorname{SINR}_{i \rightarrow j}=\frac{P h_{i j}}{B N_{0}+\sum_{k \neq i, k=1}^{n} P h_{k j}} \geq \beta,
$$

where $h_{i j}$ is the channel attenuation factor between nodes $i$ and $j$, and $B N_{0}$ is the total ambient noise power. We assume that the channel attenuation factors are completely determined by the path loss model and hence $h_{i j}=\left\|X_{i}-X_{j}\right\|^{-\alpha}$. We assume that $\beta \geq 1$ in all our analysis.

We assume that the data rate for each successful transmission is $W$ bits/second, which is a constant value and does not depend on $n$. Given that $W$ does not change the order capacity of the network, we normalize its value to one. Thus, we say that in a slot $t$ a node $i$ transmits a binary symbol $Y_{t, i} \in(0,1, ?)$, where $Y_{t, i}=$ ? (an erasure) if the node $i$ is silent. Each node recieves a symbol $Z_{t, i} \in(0,1, ?)$, where $Z_{t, i}=$ ? if the node $i$ does not satisfy the interference and/or attenuation conditions for any transmitter. If the transmission from node $j$ to node $i$ satisifes the constraints for succesful reception then $Z_{t, i}=Y_{t, j}$.

We highlight that the above model allows the broadcast of common information from a transmitter to all neighbouring recievers which satisfy the interference and attenuation conditions for successful reception. However we do not consider the case of MPT (or MPR) which allows tranmission (or reception ) of unique information to (from) multiple nodes in a single slot. Thus, our model is similar to that of citeLGT07 and [16]. In order to appropriately model NC, we assume that the information transmitted by a node can be an arbitrary function of the information previously recieved by the node.

We focus on the traffic scenario in which each node of the wireless network acts as a multicast source for a randomly chosen set of $m$ destinations.

\section{Definition 3.4: Feasible rate}

In a wireless ad hoc network with $n$ nodes in which each source transmits its packets to $m$ destinations, a throughput of $\lambda_{m}(n)$ bits per second for each multicast session is feasible if there is a spatial and temporal scheme for scheduling networkcoded transmissions, such that, by operating the network in a multi-hop fashion, coding and buffering at intermediate nodes when awaiting transmission, every source node can send $\lambda_{m}(n)$ bits per second on average to its $m$ chosen destination nodes. That is, there is a $T<\infty$ such that in every time interval $[(i-1) T, i T]$ every node can send $T \lambda_{m}(n)$ bits to its corresponding destination nodes. Let $C_{m}(n)$ represent the maximum feasible rate.

\section{Definition 3.5: Throughput Order}

$C_{m}(n)$ is said to be of order $\Theta(f(n))$ bits/second if there exist deterministic positive constants $c$ and $c^{\prime}$ such that

$$
\left\{\begin{array}{l}
\lim _{n \rightarrow \infty} \operatorname{Prob}\left(C_{m}(n)=c f(n) \text { is feasible }\right)=1 \\
\lim _{n \rightarrow \infty} \operatorname{Prob}\left(C_{m}(n)=c^{\prime} f(n) \text { is feasible }\right)<1 .
\end{array}\right.
$$

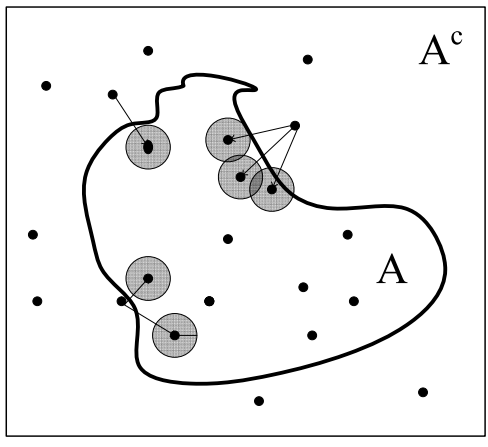

Fig. 1. Generalized Sparsity Cut

\section{Definition 3.6: Vertex Cut}

Given a node set $V$, a cut is the separation of the vertex set $V$ into two disjoint and exhaustive subsets $\left(S, S^{C}\right)$. Here, a vertex partition can be completely described by partitioning the network-area into two region $\left(A, A^{c}\right)$ as shown in Fig. 1, thus we also refer to a closed region $A$ as a cut. The cutcapacity $C(A)$ is defined as the maximum number of packets that can be transmitted from $A^{C}$ to $A$ in a single slot.

\section{Definition 3.7: Multicast Cut-Demand}

Given a cut $A$, a source node in $A^{c}$ is said to have demand across the cut iff at least one of its destination lies in $A$. 
The multicast demand $D(A)$ across the cut is defined as the total number of sources in $A^{c}$ such that there is at least one destination in the multicast group across the cut.

Definition 3.8: Sparsest Cut

We define the sparsity $\Gamma_{A}$ of cut $A$ as the ratio

$$
\Gamma_{A}=\frac{C(A)}{D(A)}
$$

Hence, the sparsest cut is given by

$$
A^{*}=\arg \min _{A} \Gamma_{A}
$$

where $A^{*}$ has the least possible sparsity, denoted as $\Gamma_{A^{*}}$.

The notion of sparsity cut has been utilized in a number of studies related to NC. The definition of Sparsity cut used in [31] is applicable only to unicast traffic [6]. We employ a more generalized definition. Meanwhile studies such as [24] define sparsity-cuts in terms of edge-cuts, i.e. in [24] a cut does not lead to a graph (vertex) seperation. We shall use the sparsity of a cut to provide an upper bound on the rate achievable under NC. It is important that the reader understands that we are employing a definition that is distinct from studies such as [24], since [24] shows that under an alternate defintion NC can exceed the bound provided by a sparsity cut.

Finally we state the well-known Chernoff Bounds [32], which shall be repeatedly used in the rest of this paper.

Lemma 3.9: Chernoff Bounds: Consider $n$ i.i.d random variables $Y_{i} \in\{0,1\}$ with $p=\operatorname{Pr}\left(Y_{i}=1\right)$. Let $Y=\sum_{i=1}^{n} Y_{i}$. Then for any $1 \geq \delta \geq 0$ and $\delta_{2} \geq 0$ we have

$$
\begin{aligned}
& \operatorname{Pr}\left(Y \leq\left(1-\delta_{1}\right) n p\right) \leq 2 e^{\frac{-\delta_{1}^{2} n p}{3}} \\
& \operatorname{Pr}\left(Y \geq\left(1+\delta_{2}\right) n p\right) \leq 2 e^{\frac{-\delta_{2}^{2} n p}{3}}
\end{aligned}
$$

\section{Bounds For Protocol Model}

It is well-known that under the conventional definition, the sparsity cut can be used to obtain an upper bound on the unicast traffic flow in a wireless network [6], [31]. In a similar way, our generalized definition provides an upper bound for multicast flows. Furthur note that the following lemma is applicable to both the protocol as well as the phsyical model.

Lemma 4.1: Let $C_{m}(n)$ be maximum multicast flow-rate in a network and let $A^{*}$ be the sparsest cut with sparsity $\Gamma_{A^{*}}$, then we have

$$
C_{m}(n) \leq \Gamma_{A^{*}} .
$$

Proof: Consider an arbitrary cut $A$ and let $S=$ $\left(s_{1}, \ldots, s_{\|S\|}\right)$ be the set of nodes in $A^{c}$ which act as a multicast source for at least one destination node in $A$. For each source $s_{i} \in S$ let $T_{i}=\left(t_{i 1}, \ldots, t_{i\left|T_{i}\right|}\right)$ be the set of its destinations in $A$. Now, lets construct a unicast problem by choosing an arbitrary node $t_{i} \in T_{i}$ as the destination for source $s_{i}$. Let $R$ be the maximum rate that can be concurrently achieved for all the unicast sources in $S$. The classical cut-set bound [Thm 14.10.1 [22]] provides an outerbound on the sumrate in terms of conditional mutual information

$$
|S| \times R \leq I\left(Y_{t}\left(A^{C}\right) ; Z_{t}(A) / Y_{t}(A)\right)
$$

where $Y_{t}\left(A^{c}\right)$ and $Z_{t}(A)$ are the random vectors consisting of transmission and reception random variables associated with nodes in $A^{c}$ and $A$ respectively. Note that, $I\left(Y_{t}\left(A^{C}\right) ; Z_{t}(A) / Y_{t}(A)\right) \leq H\left(Z_{t}(A)\right)$ and $Z_{t}(A)$ is completely determined by the succesful transmissions from $A^{c}$ to $A$. Since $Y_{i}$ and $Z_{i}$ are binary, $H\left(Z_{t}\left(A^{c}\right)\right)$ is less than the maximum number of succesful transmission from $A^{c}$ to $A$ in a single slot, i.e. $H\left(Z_{t}\left(A^{c}\right)\right) \leq C(A)$ information bits. Additionally Def. 3.7 implies that $|S|=D(A)$ and hence we have

$$
D(A) R \leq C(A)
$$

Finally note that if a multicast rate from a source to multiple destinations is feasible, then clearly it is feasible to achieve the same rate to any one arbitrarily chosen node from this set of destinations. Hence $C_{m}(n) \leq R$ impying that

$$
C_{m}(n) \leq \frac{C(A)}{D(A)}=\Gamma_{A}
$$

for any arbitrary cut $A$.

It should be highlighted that the above deductions imply that the maximum multicast flow-rate is less than the sparsity of any arbitrary cut. Thus, to obtain an upper bound on the network capacity, we are free to choose a region $A$ of any arbitrary shape and size. In this work we shall utilize cuts of square shape as shown in Fig.2, with length $L_{A}=4 l_{A}$, i.e., each side of the square $A$ has length $l_{A}$. The parameter $l_{A}$ plays a crucial role in deducing the required upper bounds. In particular, we choose $l_{A}$ so as to guarantee that the demand $D(A)=\Theta(n)$.

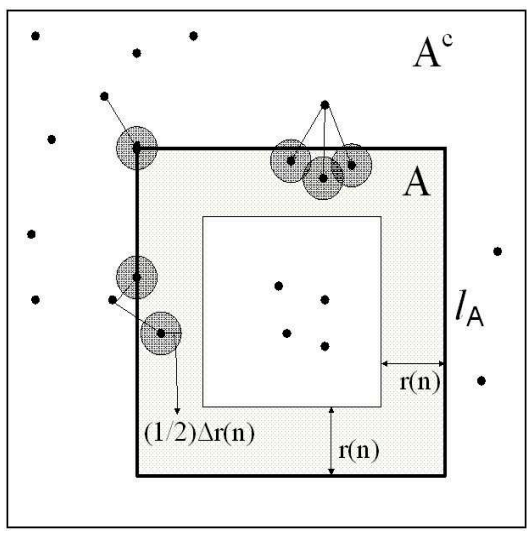

Fig. 2. Cut Capacity under Protocol Model

Lemma 4.2: In a random network with $n$ nodes, each acting as source for $m$ randomly chosen nodes, for every $\epsilon \geq 0$ if

$$
\begin{aligned}
& l_{A}=\frac{1}{\sqrt{(1+\epsilon) m}} \text { for } m \leq \frac{1}{4(1+\epsilon) r(n)^{2}} \\
& l_{A}=2 r(n) \text { for } m \geq \frac{1}{4(1+\epsilon) r(n)^{2}}
\end{aligned}
$$

then for any $1 \geq \delta_{1} \geq 0$ and $n$ such that $\frac{n}{\log (2 n)} \geq \frac{3}{\delta_{1}^{2} c_{1}}$, w.h.p we have

$$
D(A) \geq\left(1-\delta_{1}\right) n c_{1}
$$


where $c_{1}=\left(1-\frac{1}{1+\epsilon}\right)\left(1-\frac{1}{e^{\frac{1}{1+\epsilon}}}\right)$.

Proof: Let $q$ be the probability that a randomly chosen node $i$ has demand across cut $A$. Thus,

$q \quad=\operatorname{Pr}\left(i \in A^{c}\right) \times \operatorname{Pr}($ at least one destination of $i \in A)$ $\geq(1-\|A\|)\left(1-(1-\|A\|)^{m}\right)=\left(1-l_{A}^{2}\right)\left(1-\left(1-l_{A}^{2}\right)^{m}\right)$

Now, note that $\frac{1}{\sqrt{1+\epsilon}} \geq l_{A} \geq \frac{1}{\sqrt{(1+\epsilon) m}}$ for all $m$. Hence, we have

$$
\begin{array}{r}
q \geq\left(1-\frac{1}{1+\epsilon}\right)\left(1-\left(1-\frac{1}{(1+\epsilon) m}\right)^{m}\right) \\
\geq\left(1-\frac{1}{1+\epsilon}\right)\left(1-\frac{1}{e^{\frac{1}{1+\epsilon}}}\right)=c_{1}
\end{array}
$$

where the second inequality follows from the well-known fact that $e^{-x} \geq(1-x)$ for any $1 \geq x \geq 0$.

Let $Y_{i}$ be an indicator variable that is equal to one if the node $i$ has demand across cut $A$. Thus $\operatorname{Pr}\left(Y_{i}=1\right)=q$ and $D(A)=\sum_{i=1: n} Y_{i}$. Hence, the Chernoff bound of Eq. (6) from Lemma 3.9 further implies that

$$
\operatorname{Pr}\left(D(A) \leq\left(1-\delta_{1}\right) n q\right) \leq 2 e^{\frac{-\delta_{1}^{2} n q}{3}}
$$

Now $\frac{n}{\log (2 n)} \geq \frac{3}{\delta_{1}^{2} c_{1}}$ guarantees that

$$
\operatorname{Pr}\left(D(A) \leq\left(1-\delta_{1}\right) n q\right) \leq \frac{1}{n}
$$

A choice of $l_{A}=\frac{1}{\sqrt{\left(1+\epsilon_{1}\right) m}}$ can be used in the above lemma for all $m$, and such a condition would be sufficient to prove the required result that demand $D(A) \geq\left(1-\delta_{1}\right) n c_{1}$ w.h.p. However, in the following analysis we require that $l_{A} \geq 2 r(n)$. Therefore, we introduce the condition that $l_{A}=2 r(n)$ for $m \geq \frac{1}{4\left(1+\epsilon_{1}\right) r(n)^{2}}$. Note that if $m \geq \frac{1}{4\left(1+\epsilon_{1}\right) r(n)^{2}}$, then $2 r(n) \geq \frac{1}{\sqrt{\left(1+\epsilon_{1}\right) m}}$.

We invoke the following important observation to obtain an upperbound on the cut-capacity.

Remark 4.3: In [16], it was observed that in any time slot, a disk of radius $\frac{\Delta r(n)}{2}$ centered at each receiver in that slot should be disjoint. However, this fact does not apply to the case in which nodes exploit broadcast transmissions, as is done when nodes are capable of employing NC. Indeed, as shown in Fig.2, the disks can overlap if the associated nodes are receiving identical information from a common transmitter. Nevertheless, as highlighted in [5], even under the $\mathrm{NC}$ assumption, the union of the disks centered at the receivers of one transmission should be disjoint from the union of the disks centered at the receivers of another transmission.

Lemma 4.4: If a square-shaped cut $A$ has side-length $l_{A} \geq$ $2 r(n)$, then the cut capacity satisfies

$$
C(A) \leq \frac{16 L_{A}}{\pi \Delta^{2} r(n)}
$$

under the protocol model.

Proof: In the protocol model, the distance between a transmitter and a receiver is bounded by $r(n)$. Hence, any node in $A$ that receives a transmission from $A^{c}$ should lie within a distance $r(n)$ from the boundary of the cut, i.e., all the receivers must be placed within an annular region of area

$$
\begin{aligned}
& l_{A}^{2}-\left(l_{A}-2 r(n)\right)^{2} \\
= & 4 l_{A} r(n)-4 r(n)^{2} \\
\leq & 4 l_{A} r(n)=L_{A} r(n)
\end{aligned}
$$

where the length $L_{A}$ of the cut is the perimeter of the region $A$.

We observe that each transmission across the cut will not allow any more transmission within an area of at least $\frac{\pi \Delta^{2} r(n)^{2}}{4}$. Additionally, at least $\frac{1}{4}$ of this area has to fall within the annular region near the cut boundary. Therefore,

$$
\begin{aligned}
C(A) & =\text { max. no. of transmissions from } A^{c} \text { to } A \\
& \leq \frac{\text { Area of annular region }}{\frac{\pi \Delta^{2} r(n)^{2}}{4 \times 4}} \\
& =\frac{16 L_{A}}{\pi \Delta^{2} r(n)}
\end{aligned}
$$

Theorem 4.5: Under the protocol model, the multicast capacity of a random geometric network with $\mathrm{NC}$ has the following upper bound w.h.p

$$
\begin{aligned}
C_{m}(n) & =\frac{c_{2}}{\sqrt{3\left(1+\epsilon_{1}\right) m n \log (n)}} \text { if } \mathrm{m} \leq \frac{n\left(1+\epsilon_{1}\right)^{-1}}{12 \log (n)} \\
C_{m}(n) & =\frac{2 c_{2}}{n} \text { if } \mathrm{m} \geq \frac{n\left(1+\epsilon_{1}\right)^{-1}}{12 \log (n)}
\end{aligned}
$$

when $\frac{n}{\log (2 n)} \geq \frac{3}{\delta_{1}^{2} c_{1}}$, where $c_{2}=\frac{64\left(1+\epsilon_{1}\right) e^{\frac{1}{1+\epsilon_{1}}}}{\pi \Delta^{2} \epsilon_{1}\left(1-\delta_{1}\right)\left(e^{\frac{1}{1+\epsilon_{1}}}-1\right)}$ and $\delta_{1}, \epsilon_{1} \geq 0$

Proof: On account of Lemma 4.1, we can obtain an upper bound on the network capacity by just providing a bound for the sparsity $\Gamma_{A}$. Furthermore note that $L_{A}=4 l_{A}$, and hence due to Lemma 4.4 we can say that for all $l_{a} \geq 2 r(n)$ we have

$$
C_{m}(n) \leq \frac{64 l_{A}}{\pi \Delta^{2} r(n) D(A)} .
$$

Consider $m \geq \frac{1}{4\left(1+\epsilon_{1}\right) r(n)^{2}}$. If we choose $l_{A}=2 r(n)$, then from Lemma 4.2 we have that for all $n$ such that $\frac{n}{\log (2 n)} \geq$ $\frac{3}{\delta_{1}^{2} c_{1}}$ w.h.p. we have $D(A) \geq\left(1-\delta_{1}\right) n c_{1}$. Therefore,

$$
C_{m}(n) \leq \frac{128}{\pi \Delta^{2}\left(1-\delta_{1}\right) n c_{1}}
$$

Similarly, if we choose $l_{A}=\frac{1}{\sqrt{(1+\epsilon) m}}$ for all $m \leq$ $\frac{1}{4\left(1+\epsilon_{1}\right) r(n)^{2}}$, we have

$$
C_{m}(n) \leq \frac{64 \frac{1}{\sqrt{\left(1+\epsilon_{1}\right) m}}}{\pi \Delta^{2}\left(1-\delta_{1}\right) r(n) n c_{1}} .
$$

Note that, for all $m \leq \frac{1}{4\left(1+\epsilon_{1}\right) r(n)^{2}}, C_{m}(n)$ is maximized by choosing the smallest possible value of $r(n)$. Nevertheless the Connectivity Criteria (Lemma 3.2) requires that $r(n) \geq$ 
$\sqrt{\frac{3 \log (n)}{n}}$. The final result is obtained by substituting the value of $c_{1}$ and $r(n)=\sqrt{\frac{3 \log (n)}{n}}$ in Eqs. 21-22.

The multicast capacity under pure routing has been characterized in [13], [14].

Theorem 4.6: [13], [14] Under the protocol model, the multicast capacity of a random geometric network with routing has a tight bound of

$$
\begin{aligned}
& C_{m}(n)=\Theta\left(\frac{1}{\sqrt{m n \log (n)}}\right) \text { if } m=O\left(\frac{n}{\log (n)}\right) \\
& C_{m}(n) \quad=\Theta\left(\frac{1}{n}\right) \text { if } m=\Omega\left(\frac{n}{\log (n)}\right)
\end{aligned}
$$

Network coding (NC) is a generalization of routing and thus any capacity achieved by routing is necessarily achieved by NC. Hence,

Theorem 4.7: Under the protocol model, the multicast capacity of a random geometric network with $\mathrm{NC}$ has a tight bound equal to

$$
\begin{aligned}
& C_{m}(n)=\Theta\left(\frac{1}{\sqrt{m n \log (n)}}\right) \text { if } m=O\left(\frac{n}{\log (n)}\right) \\
& C_{m}(n) \quad=\Theta\left(\frac{1}{n}\right) \text { if } m=\Omega\left(\frac{n}{\log (n)}\right)
\end{aligned}
$$

Finally, we can arrive at the following conclusion.

Corollary 4.8: The multicast throughput order gain provided by $\mathrm{NC}$ over routing in a random geometric network is $O(1)$ under the protocol model.

\section{BOUNDS FOR PHySICAL MODEL}

In order to prove the upper bound under the physical model we utilize a circular cut, instead of square shaped cut, with radius $r_{A}$ as shown in Fig. 3. Additionally, we utilize the following property of the physical model. A similar property of "straight-lined cuts" has also been utilized by Liu, et. al. [6].

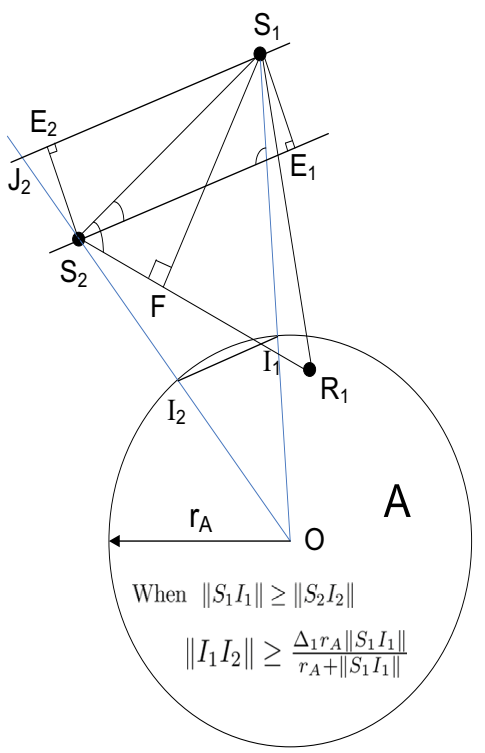

Fig. 3. Geometric property of transmissions across the cut
Lemma 5.1: Consider a circular cut $A$ of radius $r_{A}$ with its center at point $O$. Let $S_{1}$ and $S_{2}$ be two nodes outside $A$ transmitting across the cut in the same slot. We claim that the arc subtended by angle $\angle S_{1} O S_{2}$ on cut $A$ has a length of at least

$$
\frac{\Delta_{1} r_{A} \max \left\{L_{1}, L_{2}\right\}}{r_{A}+\max \left\{L_{1}, L_{2}\right\}}
$$

where $\Delta_{1}=\left(\beta^{\frac{1}{\alpha}}-1\right)$ and $L_{i}$ represents the (minimum) distance of transmitter $S_{i}$ from cut $A$.

Proof: Without loss of generality we can assume that $S_{1}$, $S_{2}$ are placed as shown in Fig. 3 and $L_{1} \geq L_{2}$. In Fig. 3 the rays $O S_{1}$ and $O S_{2}$ intersect the cut $A$ at $I_{1}$ and $I_{2}$ respectively. Therefore, $L_{1}=\left\|S_{1} I_{1}\right\|$ and $L_{2}=\left\|S_{2} I_{2}\right\|$. Furthermore, the length of segment $I_{1} I_{2}$ is less than the length of the arc subtended by $\angle S_{1} O S_{2}$. Hence, in order to prove the claim, it is sufficient to show that

$$
\left\|I_{1} I_{2}\right\| \geq \frac{\Delta_{1} r_{A}\left\|S_{1} I_{1}\right\|}{r_{A}+\left\|S_{1} I_{1}\right\|}
$$

Consider a receiver $R_{1}$ that lies inside $A$ and can successfully decode a transmission from $S_{1}$. It follows from Eq. 2 in Definition 3.3 that

$$
\begin{aligned}
& \frac{P\left\|S_{1} R_{1}\right\|^{-\alpha}}{B N_{o}+P\left\|S_{2} R_{1}\right\|^{-\alpha}} \geq \beta \\
& \Longrightarrow\left\|S_{2} R_{1}\right\| \geq \beta^{\frac{1}{\alpha}}\left\|S_{1} R_{1}\right\|=\left(1+\Delta_{1}\right)\left\|S_{1} R_{1}\right\|
\end{aligned}
$$

Consider the triangle formed by $S_{1}, S_{2}$ and $R_{1}$, as shown in Fig. 3. Now draw a perpendicular from $S_{1}$ to $F$, which is a point on segment $S_{2} R_{1}$. Note that $\left\|F R_{1}\right\| \leq\left\|S_{1} R_{1}\right\|$ and hence it is easy to show that $\left\|S_{2} F\right\| \geq \Delta_{1}\left|S_{1} R_{1}\right|$. Now draw a line through $S_{2}$ parallel to segment $I_{1} I_{2}$ and drop a perpendicular $S_{1} E_{1}$ on this line. Since $\angle S_{1} S_{2} E_{1} \leq \angle S_{1} S_{2} R_{1}$, we have $\cos \left(\angle S_{1} S_{2} E_{1}\right) \geq \cos \left(\angle S_{1} S_{2} R_{1}\right)$, which implies that $\left|S_{2} E_{1}\right| \geq\left|S_{2} F\right|$. Similarly draw a line through $S_{1}$ parallel to $I_{1} I_{2}$. Let this line intersect the ray $O S_{2}$ at $J_{2}$. Drop a perpendicular $S_{2} E_{2}$ on line $S_{1} J_{2}$. Since the triangle $S_{1} O J_{2}$ is isosceles, $\angle S_{1} J_{2} S_{2}$ is acute and hence $E_{2}$ should lie within the segment $S_{1} J_{2}$. Hence, $\left\|S_{1} J_{2}\right\| \geq\left\|S_{1} E_{2}\right\|$. Since $S_{2} E_{1} S_{1} E_{2}$ forms a rectangle we get $\left\|S_{1} J_{2}\right\| \geq \Delta_{1}\left|S_{1} R_{1}\right|$. Finally, we note that $\left\|S_{1} R_{1}\right\| \geq\left\|S_{1} I_{1}\right\|$ because $S_{1} I_{1}$ is the shortest distance between $S_{1}$ and circle $A$. Hence,

$$
\left\|S_{1} J_{2}\right\| \geq \Delta_{1}\left\|S_{1} I_{1}\right\|
$$

Consider the triangle $O S_{1} J_{2}$. The Basic Proportionality Theorem implies that

$$
\left\|I_{1} I_{2}\right\|=\frac{\left\|S_{1} J_{2}\right\|\left\|O I_{1}\right\|}{\left\|O S_{1}\right\|}
$$

Substituting Eq. 30 in Eq. 31 proves the claim in Eq. 28

Theorem 5.2: Under the physical model, the multicast capacity of a random geometric network with NC has the following upper bound w.h.p

$$
C_{m}(n)=O\left(\frac{1}{\sqrt{m n}}\right),
$$


when $m=O\left(\frac{n}{\log (n)^{2}}\right)$ and $n \rightarrow \infty$.

Proof: Consider a circular cut $A$ with radius $r_{A}=\frac{1}{4 \sqrt{m}}$. Divide the region $A^{c}$, as shown in Fig. 4, into sub-region $B$ and $A^{c}-B$, where the $B$ is an annular region of width $\frac{1}{\sqrt{n}}$. Let $n_{B}$ and $n_{A^{C}-B}$ be the maximum number of nodes, from region $B$ and region $A^{c}-B$ respectively, that can transmit to region $A$ in a single time slot. Hence,

$$
C(A) \leq n_{B}+n_{A^{c}-B}
$$

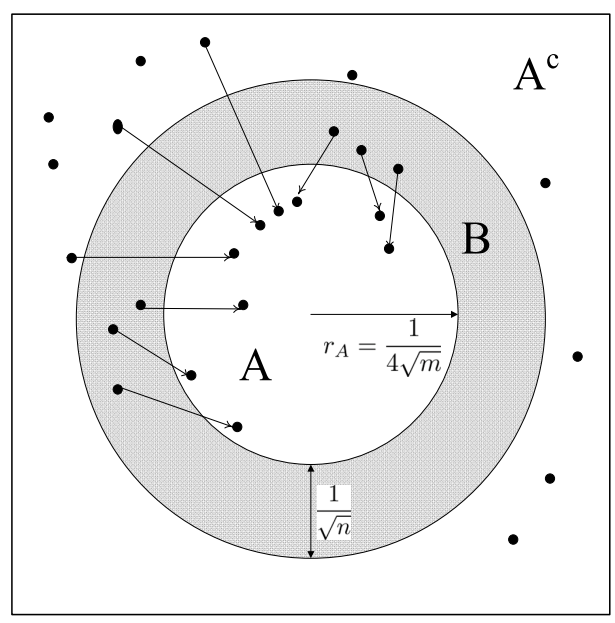

Fig. 4. Cut Capacity under Physical Model

A transmission from any node in region $A^{c}-B$ to any node in region $A$ has a minimum hop-length of $\frac{1}{\sqrt{n}}$. Consequently, Lemma 5.1 implies that any two transmitters in $A^{c}-B$, that transmit in the same slot, have to be separated such that they subtend an arc on $A$ of length at least $\frac{\Delta_{1} r_{A} \frac{1}{\sqrt{n}}}{r_{A}+\frac{1}{\sqrt{n}}}$. Since the circumference of $A$ is $2 \pi r_{A}$ we have

$$
\begin{aligned}
n_{A^{c}-B} & \leq 2 \pi r_{A} \times \frac{r_{A}+\frac{1}{\sqrt{n}}}{\Delta_{1} r_{A} \frac{1}{\sqrt{n}}} \\
& =\frac{2 \pi}{\Delta_{1}}\left(\frac{\sqrt{n}}{4 \sqrt{m}}+1\right) \leq \frac{5 \pi \sqrt{n}}{2 \Delta_{1} \sqrt{m}}
\end{aligned}
$$

To obtain a bound on $n_{B}$, observe that the area of region $B$ is given by

$$
\begin{aligned}
|B| & =\pi\left(r_{A}+\frac{1}{\sqrt{n}}\right)^{2}-\pi r_{A}^{2} \\
& =\frac{2 \pi r_{A}}{\sqrt{n}}+\frac{\pi}{n} \leq \frac{\pi}{2 \sqrt{m n}}+\frac{\pi}{\sqrt{m n}} \\
& \leq \frac{3 \pi}{2 \sqrt{m n}}
\end{aligned}
$$

If $m=O\left(\frac{n}{(\log (n))^{2}}\right)$, there exists a constant $c_{3} \geq 0$ such that

$$
|B| \leq \frac{c_{3} \log (n)}{n}
$$

The total number of nodes in $B$ is necessarily greater than $n_{B}$. Therefore, the Chernoff Bound of Eq. 6 implies that, for any $\delta_{2} \geq 0$, we have

$$
\begin{aligned}
& \operatorname{Pr}\left(n_{B} \leq \frac{3 \pi\left(1+\delta_{2}\right) \sqrt{n}}{2 \sqrt{m}}\right) \leq 2 e^{\frac{-\delta_{2}^{2} n|B|}{3}} \\
\leq & 2 e^{\frac{-\delta_{2}^{2} \log (n)}{3 c_{3}}}=\frac{2}{n^{\frac{\delta_{2}^{2}}{3 c_{3}}}} .
\end{aligned}
$$

Consequently, if we choose $\delta_{2} \geq 3 c_{3}$, then as $n \rightarrow \infty$ w.h.p we have

$$
\begin{array}{r}
C(A) \leq \frac{3 \pi\left(1+\delta_{2}\right) \sqrt{n}}{2 \sqrt{m}}+\frac{5 \pi \sqrt{n}}{2 \Delta_{1} \sqrt{m}} \\
=\frac{\pi\left(3\left(1+\delta_{2}\right) \Delta_{1}+5\right) \sqrt{n}}{2 \Delta_{1} \sqrt{m}}
\end{array}
$$

In the previous section, we have already shown that w.h.p the demand across square shaped cut with area $O\left(\frac{1}{m}\right)$ is of the order of $\Theta(n)$. Such a property is valid for circular cuts also. Let $q_{1}$ be probablity that a source node in $A^{c}$ has at least one of its $m$ destinations in the circle $A$. We can show that

$$
\begin{aligned}
q_{1} & \geq\left(1-\frac{1}{16}\right)\left(1-\left(1-\frac{1}{16 m}\right)^{m}\right) \\
& =\frac{15\left(1-e^{\frac{1}{16}}\right)}{16}=c_{4}
\end{aligned}
$$

The Chernoff Bound of Eq. 7 implies that there exists a $1 \geq$ $\delta_{1} \geq 0$ such that as $n \rightarrow \infty$ w.h.p. $D(A) \geq\left(1-\delta_{1}\right) c_{4} n$. Therefore, the Sparsity bound from Lemma 4.1, along with Eqs. 38 and 39 implies that w.h.p.

$$
C_{m}(n) \leq\left(\frac{\pi\left(3\left(1+\delta_{2}\right) \Delta_{1}+5\right)}{2 \Delta_{1}\left(1-\delta_{1}\right) c_{4}}\right) \frac{1}{\sqrt{m n}}
$$

The mathematical techniques used in the above proof cannot be utilized to obtain a upper bound on multicast capacity of NC for all values of $m$. In particular note that Eq. 36 and hence the convergence condition in Eq. 37 requires that $m=$ $O\left(\frac{n}{(\log (n))^{2}}\right)$. Therefore, we consider an alternative approach to obtain upperbounds. This approach shall give us a tighter upper bound for $m=\Omega\left(\frac{n}{\log (n)}\right)$.

Theorem 5.3: Under the physical model, the multicast capacity in a random geometric network with $\mathrm{NC}$ has the following upper bound w.h.p.

$$
\begin{aligned}
& C_{m}(n)=O\left(\frac{1}{m \log (n)}\right) \text { if } m \leq \frac{n}{\log (n)} \\
& C_{m}(n)=O\left(\frac{1}{n}\right) \text { if } m \geq \frac{n}{\log (n)}
\end{aligned}
$$

Proof: Decompose the network into squarelets of sidelength $\sqrt{\frac{\log (n)}{9 n}}$. Let $J$ be an event that there exists a squarelet containing at least $\frac{\left(1-\delta_{3}\right) \log (n)}{9 n}$ nodes, where $1 \geq \delta_{3} \geq 0$, with all its eight adjoining squarelets empty. The event $J$ is illustrated in Fig. 5. We are interested in showing that the event $J$ occurs w.h.p. Let $\eta$ represent the total number of nodes in a 
squarelet, $p_{1}=\operatorname{Pr}(\eta=0)$ and $p_{2}=\operatorname{Pr}\left(\eta \leq \frac{\left(1-\delta_{3}\right) \log (n)}{9 n}\right)$, where $1 \geq \delta_{3} \geq 0$. $p_{1}$ can be computed as

$$
p_{1}=\left(1-\frac{\log (n)}{9 n}\right)^{n}=e^{\frac{-\log (n)}{9}}=n^{\frac{-1}{9}} .
$$

We used the fact that $\lim _{n \rightarrow \infty}\left(1-\frac{a}{n}\right)^{n}=e^{-a}$.

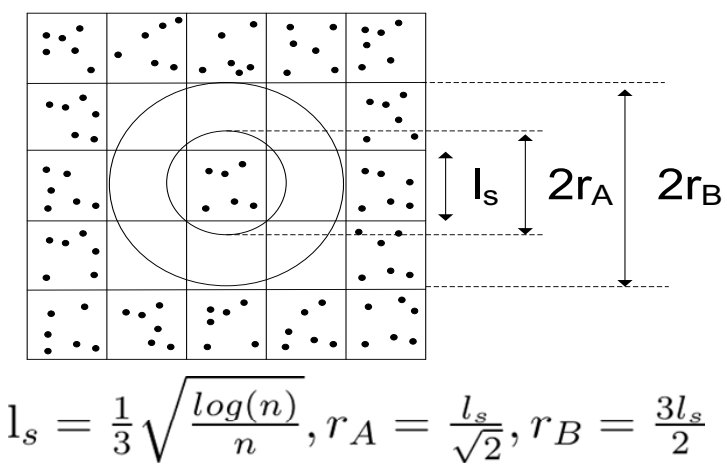

Fig. 5. Clustering of nodes

In addition, Eq. 6 implies that

$$
\begin{aligned}
p_{2} \quad & \operatorname{Pr}\left(\eta \leq \frac{\left(1-\delta_{3}\right) \log (n)}{9 n}\right) \\
& \leq 2 e^{\frac{-\delta_{3}^{2} \log (n)}{27}}=2 n^{\frac{-\delta_{3}^{2}}{27}} .
\end{aligned}
$$

Therefore, as $n \rightarrow \infty$, in the limit we have

$$
\begin{aligned}
\operatorname{Pr}(J) & \geq 1-\left(1-\left(1-p_{2}\right) p_{1}^{8}\right)^{\frac{9 n}{\log (n)}} \\
& \geq 1-\left(1-\left(1-2 n^{\frac{-\delta_{3}^{2}}{27}}\right) n^{\frac{-8}{9}}\right)^{\frac{9 n}{\log (n)}} \\
& \geq 1-\left(\left(1-\frac{n^{\frac{1}{9}}\left(1-2 n^{\frac{-1}{27}}\right)}{n}\right)^{n}\right)^{\frac{9}{\log (n)}} \\
& =1-e^{-9 \frac{n^{\frac{1}{9}}\left(1-2 n \frac{-1}{27}\right)}{\log (n)}}=1 .
\end{aligned}
$$

Note that $e^{-9 \frac{n \frac{1}{9}\left(1-2 n-\frac{1}{27}\right)}{\log (n)}}$ approaches zero faster than $\frac{1}{n}$ when $n \rightarrow \infty$.

Let us choose a circular cut $A$ of radius $r_{A}=\frac{l_{s}}{\sqrt{2}}$ such that $A$ circumscribes a squarelet satisfying property $J$. Observe that we can draw another circle $B$ of radius $r_{B}=\frac{3 l_{s}}{2}$ concentric to $A$, such that all nodes that transmit across the cut $A$ are placed outside $B$. Therefore the minimum hop-length of any transmission across the cut $A$ is atleast $r_{B}-r_{A}$. Therefore Lemma 5.1 implies that

$$
\begin{aligned}
C(A) & \leq 2 \pi r_{A} \times \frac{r_{A}+\left(r_{B}-r_{A}\right)}{\Delta_{1} r_{A}\left(r_{B}-r_{A}\right)}=\frac{2 \pi r_{A}}{\Delta_{1}\left(r_{B}-r_{A}\right)} \\
& =\frac{2 \pi \frac{l_{s}}{\sqrt{2}}}{\Delta_{1}\left(\frac{3 l_{s}}{2}-\frac{l_{s}}{\sqrt{2}}\right)}=\frac{\pi 2 \sqrt{2}}{\Delta_{1}(3-\sqrt{2})}=c_{5}
\end{aligned}
$$

Now let $p_{3}$ be the probability that a source has demand across cut $A$. Observe that all the nodes inside the circle $A$ are within the middle squarelet. Hence the Chernoff Bound can be used to show that as $n \rightarrow \infty$ w.h.p the total number of nodes outside the circle $A$ are at least $n-\frac{\left(1+\delta_{4}\right) \log (n)}{9}$, where $\delta_{4} \geq 0$. Therefore, as $n \rightarrow \infty$ w.h.p.,

$$
\begin{aligned}
p_{3}=\left(1-\frac{\left(1+\delta_{4}\right) \log (9 n)}{n}\right)\left(1-\left(1-\frac{\left(1-\delta_{3}\right) \log (n)}{9 n}\right)^{m}\right) \\
=\left(1-e^{\frac{-m\left(1-\delta_{3}\right) \log (n)}{9 n}}\right)
\end{aligned}
$$

In the above equation we have $p_{3}=\Theta(1)$ when $m=$ $\Omega\left(\frac{n}{\log (n)}\right)$, while when $m=O\left(\frac{n}{\log (n)}\right)$ we have that

$$
p_{3} \geq \frac{m\left(1-\delta_{3}\right) \log (n)}{9 n}
$$

Therefore, an application of Eq. 7 allows us to show that $D(A)=\Omega(m \log (n))$ when $m=O\left(\frac{n}{\log (n)}\right)$, while $D(A)=$ $\Omega(n)$ when $m=\Omega\left(\frac{n}{\log (n)}\right)$. We get the final result by calculating the sparsity $\Gamma_{A}=\frac{C(A)}{D(A)}$ which, as established by Lemma 4.1 provides an upperbound for the capacity $C_{m}(n)$.

The upper bounds stated in the above theorem are identical to those of Theorem 2 in [15] and the initial steps in our proof are similar to those in [15]. However, we highlight that our eventual argument utilizes the geometric properties of the cut and hence is distinct from [15]. In particular, the claims and the proof in [15] is applicable only to routing, while our bounds apply to NC.

Keshavarz et. al. [15] have established the following lower bound on the multicast capacity under routing.

Theorem 5.4: Under the physical model, the multicast capacity of a random geometric network with routing has the following lower bound w.h.p.

$$
\begin{array}{lcl}
C_{m}(n) & =\Omega\left(\frac{1}{\sqrt{m n}}\right) & \text { if } m \leq \frac{n}{\log (n)^{3}} \\
C_{m}(n)=\Omega\left(\frac{1}{m \sqrt{\log (n)^{3}}}\right) & \text { if } \frac{n}{\log (n)^{3}} \leq m \leq \frac{n}{\log (n)^{2}}(59) \\
C_{m}(n)=\Omega\left(\frac{1}{\sqrt{m n \log (n)}}\right) & \text { if } \frac{n}{\log (n)^{2}} \leq m \leq \frac{n}{\log (n)}(51) \\
C_{m}(n) & =\Omega\left(\frac{1}{n}\right) \text { if } & \frac{n}{\log (n)} \leq m
\end{array}
$$

Given that any capacity achieved by routing is necessarily achievable by NC, putting together the deductions up to this point, we arrive at the following result.

Theorem 5.5: Under the physical model, the multicast capacity in a random geometric network with $\mathrm{NC}$ has a tight bound w.h.p. of

$$
\begin{aligned}
& C_{m}(n)=\Theta\left(\frac{1}{\sqrt{m n}}\right) \text { if } m \leq \frac{n}{\log (n)^{3}} \\
& C_{m}(n) \quad=\Theta\left(\frac{1}{n}\right) \text { if } \frac{n}{\log (n)} \leq m .
\end{aligned}
$$

Consequently,

Corollary 5.6: In a random geometric network with $n$ nodes and for values of $m \leq \frac{n}{\log (n)^{3}}$ and $\frac{n}{\log (n)} \leq m$, the 
multicast throughput order gain provided by $\mathrm{NC}$ over routing is $O(1)$ under the physical model.

\section{CONCLUSION}

Network coding (NC) has received considerable attention, and recent results for specific instantiations of $\mathrm{NC}$ have led many to infer that $\mathrm{NC}$ could lead to order throughput gains for multicasting in wireless networks. In this work, we used the protocol and physical models to show that the order throughput gain derived from $\mathrm{NC}$ for multicasting and broadcasting in wireless networks is bounded by a constant. That is, as the network size increases, NC renders the same order throughput as traditional store-and-forward routing. However for values of $\frac{n}{\log (n)^{3}} \leq m \leq \frac{n}{\log (n)}$, there is a gap between NC and traditional routing. It is the subject of future work to investigate whether this gap can be closed (see Fig. 6).

Despite this negative result on order throughput for $\mathrm{NC}$, constant-factor gains should not be ignored in practice, and $\mathrm{NC}$ may still prove to have much utility in wireless networks.

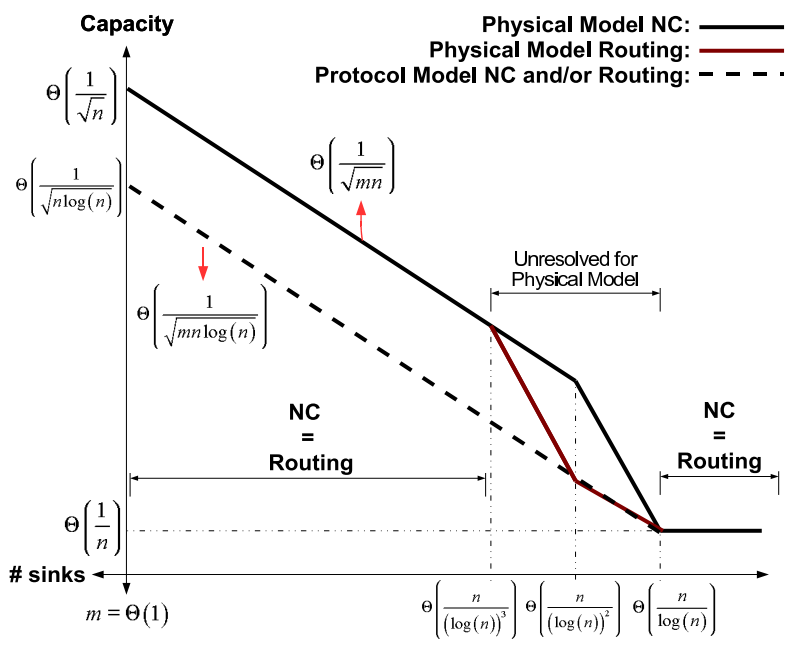

Fig. 6. Throughput Order of Network Coding

\section{REFERENCES}

[1] R. W. Yeung and Z. Zhang, "Distributed source coding for satellite communications," IEEE Transactions on Information Theory, vol. 45 , no. 4, p. $11111120,1999$.

[2] R. Ahlswede, C. Ning, S.-Y. R. Li, and R. W. Yeung, "Network information flow," IEEE Transactions on Information Theory, vol. 46, no. 4, pp. 1204-1216, 2000.

[3] S. Katti, H. Rahul, W. Hu, D. Katabi, M. Medard, and J. Crowcroft, "Xors in the air: Practical wireless network coding," in ACM SIGCOMM 2006, Pisa, Italy., September 2006.

[4] S. Chachulski, M. Jennings, S. Katti, and D. Katabi, "Trading structure for randomness in wireless opportunistic routing," in ACM SIGCOMM 2007, Kyoto, Japan., August 2007.

[5] J. Liu, D. Goeckel, and D. Towsley, "The throughput order of ad hoc networks employing network coding and broadcasting," in Proc. of IEEE MILCOM 2006, Washington DC, Alaska, USA., October 23-25 2006.

[6] — "Bounds on the gain of network coding and broadcasting in wireless networks," in Proc. of IEEE INFOCOM 2007, Anchorage, Alaska, USA., May 6-12 2007.

[7] A. Keshavarz-Haddad and R. Riedi, "Bounds on the benefit of network coding: Throughput and energy saving in wireless networks," in Infocom 2008, Phoenix, Arizona, USA, April 2008
[8] B. Tavli, "Broadcast capacity of wireless networks," IEEE Communications Letters, vol. 10, no. 2, pp. 68-69, 2006.

[9] R. Zheng, "Information dissemination in power-constrained wireless networks," in Proc. of IEEE INFOCOM 2006, Barcelona, Catalunya, Spain, April 23-29 2006

[10] A. Keshavarz, V. Ribeiro, and R. Riedi, "Broadcast capacity in multihop wireless networks," in Proc. of ACM MobiCom 2006, Los Angeles, California, USA., September 23-29 2006.

[11] P. Jacquet and G. Rodolakis, "Multicast scaling properties in massively dense ad hoc networks," in Proc. of IEEE ICPADS 2005, Fukuoka, Japan, July 20-22 2005.

[12] S. Shakkottai, X. Liu, and R. Srikant, "The multicast capacity of wireless ad-hoc networks," in Proc. of ACM MobiHoc 2007, Montreal, Canada, September 9-14 2007

[13] X.-Y. Li, S.-J. Tang, and O. Frieder, "Multicast capacity for large scale wireless ad hoc networks," in Proc. of ACM MobiCom 2007, Montreal, Canada, September 9-14 2007.

[14] Z. Wang, H. R. Sadjadpour, and J. J. Garcia-Luna-Aceves, "A unifying perspective on the capacity of wireless ad hoc networks," in IEEE INFOCOM 2008, Phoenix, Arizona, USA., April 13-18 2008.

[15] A. Keshavarz-Haddad and R. Riedi, "Multicast capacity of large homogeneous multihop wireless networks," in IEEE WiOpt 2008, Berlin, Germany, April 2008.

[16] P. Gupta and P. R. Kumar, "The capacity of wireless networks," IEEE Transactions on Information Theory, vol. 46, no. 2, pp. 388-404, 2000

[17] M. Franceschetti, O. Dousse, D. Tse, and P. Thiran, "Closing the gap in the capacity of wireless networks via percolation theory," IEEE Transactions on Information Theory, vol. 53, no. 3, pp. 1009-1018, 2007.

[18] S. Li, Y. Liu, and X.-Y. Li, "Capacity of large wireless networks under gaussian channel model," in Proc. of ACM MobiCom 2008, San Francisco, CA, USA, September 14-19 2008.

[19] Z. Li and B. Li, "Network coding in undirected networks," in Proc. of CISS 2004, Princeton, NJ, USA., March 17-19 2004.

[20] Z. Li, B. Li, and L. Lau, "On achieving maximum multicast throughput in undirected networks," IEEE/ACM Transactions on Special issue on Networking and Information theory, vol. 52, pp. 2467-2485, 2006.

[21] S. P. Borade, "Network information flow: Limits and achievability," in IEEE International Symposium on Information Theory, July 2008.

[22] T. M. Cover and J. A. Thomas, Elements of Information Theory. Wiley, 1991.

[23] G. Kramer and S. A. Savari, "Edge-cut bounds on network coding rates," Journal of Network and Systems Management, March 2006.

[24] N. J. A. Harvey, R. Kleinberg, and A. R. Lehman, "On capacity of information networks," IEEE/ACM Transactions on Networking, June 2006.

[25] Z. Wang, S. Karande, H. R. Sadjadpour, and J. J. Garcia-Luna-Aceves, "On the capacity improvement of multicast traffic with network coding," in Proc. of IEEE MILCOM 2008, San Diego, CA, USA, November $17-$ 192008

[26] S. Zhang, S. Liew, and P. P. Lam, "Hot topic: Physical-layer network coding," in Proc. of ACM MobiCom 2006, Los Angeles, California, USA., September 23-29 2006.

[27] S. Katti, S. Gollakota, and D. Katabi, "Embracing wireless interference: Analog network coding," in Proc. of ACM SIGCOMM 2007, Kyoto, Japan, August 27-31 2007.

[28] J. J. Garcia-Luna-Aceves, H. R. Sadjadpour, and Z. Wang, "Challenges: Towards truly scalable ad hoc networks," in Proc. of ACM MobiCom 2007, Montreal, Quebec, Canada, September 9-14 2007.

[29] S. Karande, Z. Wang, H. R. Sadjadpour, and J. J. Garcia-Luna-Aceves, "Optimal scaling of multicommodity flows in wireless ad-hoc networks: Beyond the gupta-kumar barrier," in Proc. of IEEE MASS 2008, Atlanta, Georgia, USA, October 2008

[30] S. Kulkarni and P. Viswanath, "A deterministic approach to throughput scaling wireless networks," IEEE Transactions on Information Theory, vol. 50, no. 6, pp. 1041-1049, 2004.

[31] T. Leighton and S. Rao, "Multicommodity max-flow min-cut theorems and their use in designing approximation algorithms," Journal of the ACM, vol. 46, no. 6, pp. 787-832, 1999.

[32] R. Motwani and P. Raghavan, Randomized Algorithms. Cambridge University Press, 1995. 